\title{
Differential Expression of Genes Involved in Sucrose Synthesis in Source and Sink Organs of Cassava Plants Undergoing Seasonal Drought Stress
}

\author{
Supatcharee Netrphan ${ }^{1}$, Kessarin Tungngoen $^{2}$, Malinee Suksangpanomrung ${ }^{1}$, Opas Boonseng ${ }^{3} \&$ \\ Jarunya Narangajavana, ${ }^{2,4}$ \\ ${ }^{1}$ National Center for Genetic Engineering and Biotechnology, Thailand \\ ${ }^{2}$ Department of Biotechnology, Faculty of Science, Mahidol University, Thailand \\ ${ }^{3}$ Rayong Field Crops Research Center, Department of Agriculture, Thailand \\ ${ }^{4}$ Center for Cassava Molecular Biotechnology, Faculty of Science, Mahidol University, Thailand \\ Correspondence: Jarunya Narangajavana, Department of Biotechnology, Faculty of Science, Mahidol University, \\ Rama VI Road, Bangkok 10400, Thailand. Tel: 66-2-201-5319. E-mail: scjnr@mahidol.ac.th
}

Received: July 18, 2012 Accepted: August 13, 2012 Online Published: October 12, 2012

doi:10.5539/jas.v4n11p171 URL: http://dx.doi.org/10.5539/jas.v4n11p171

\begin{abstract}
Sucrose functions as a regulator of gene expression allowing plants to respond to cellular energy demands during both normal and stress conditions. Previous studies indicated that drought followed with onset of rain could negatively affect yield of starch in storage roots of cassava. In this study, molecular approaches were conducted to investigate expression profiling of sucrose phosphate synthase (SPS), sucrose phosphatase (SPP) and 14-3-3, which are importance as regulatory points to modulate availability of sucrose in cassava. In source organs, expression of cassava (Manihot esculenta Crantz.) sucrose phosphate synthase (MeSPS) and cassava sucrose phosphatase $(M e S P P)$ varied depending on developmental stage and day/night cycle. In sink organ, the levels of MeSPS, MeSPP and Me14-3-3 transcripts were affected to a much greater degree by different planting season than by root developmental stage. In response to fluctuating level of rainfall, a correlation between MeSPS and Me14-3-3 expression patterns was observed. This finding suggests the role of these enzymes and the importance of sucrose metabolism in seasonal drought stress response in cassava. Given the identified new role for cassava as an important biofuel crop as well as its current usage as a food crop, understanding on the most appropriate time to plant and harvest cassava in order to maximize starch quantity and quality is warranted.
\end{abstract}

Keywords: cassava, sucrose phosphate synthase, sucrose phosphatase, 14-3-3 protein, storage root, seasonal drought

\section{Introduction}

Cassava (Manihot esculenta Crantz) is a perennial crop of major global importance, particularly in the developing world. The plant is grown for the underground tuberous storage roots, in which nearly $85 \%$ of its total dry weight is made up of starch (Tonukari, 2004). Compared with other starchy crops, cassava has relatively long growing cycle. Generally, it takes at least 7-8 months before storage roots could be harvested.

In Thailand, cassava is generally planted twice a year; in the beginning (from the end of February to the middle of June) and at the end of rainy season (from early October to early December), with its main growing area located in the eastern (i.e., Rayong province) and northeastern regions. Given that Thailand is located in the tropical zone, the average monthly temperature does not fluctuate widely throughout the year. This is in contrast with the average rainfall level, which could range from $72 \mathrm{~mm}$ to $1,418 \mathrm{~mm}$ during dry (November-April) and rainy (May-October) season respectively (data obtained from the Thai Meteorological Department, Ministry of Information and Communication Technology, Thailand). By observing that time and conditions at harvest had an impact on cassava starch properties, Sriroth et al. (1999) recommended that starch should be extracted from roots at either early or very late developmental stage. This was investigated further and the results indicated that changes in starch properties were mainly affected by drought, particularly when it was followed with the onset of rain (Santisopasri et al., 2001; Sriroth et al., 2001). To assure starch quality, Santisopasri et al. (2001) therefore 
suggested that cassava should be planted at the start rather than at the end of rainy season. However, it is not yet clear how drought and water availability affect quality of cassava starch. To investigate into this matter, molecular approaches have been conducted to determine expression patterns of genes that might vary according to seasonal change in rainfall level.

When plants experience water stress, activation of sucrose phosphate synthase (SPS; EC 2.4.1.14), a key enzyme in sucrose synthesis, by reversible protein phosphorylation occurs, thus increasing the rate of sucrose synthesis (Quick et al., 1989). Sucrose-6-phosphate derived from this reaction is then cleaved by sucrose phosphatase (SPP, EC 3.1.3.24), which has been proposed to form a multi-protein complex with SPS (Echeverria et al., 1997). Moreover, the Ser-229 of spinach SPS has been shown to directly interact with 14-3-3 proteins in a phosphorylation and $\mathrm{Mg}^{2+}$-dependent manner (Moorhead et al., 1999; Toroser et al., 1998). Although several studies indicated that binding of 14-3-3s generally inhibited SPS activity (Szopa et al., 2001; Toroser et al., 1998; Zuk et al., 2003), there was a report on partial activation of SPS after 14-3-3 binding (Moorhead et al., 1999).

Given the importance of SPS, SPP and 14-3-3 as regulatory points to modulate availability of sucrose, genes coding for SPS, SPP and 14-3-3 proteins have been cloned from various plants and experiments on protein-protein interaction have been carried out (Bornke, 2005; Castleden et al., 2004; Chen et al., 2005). However, none of these had been conducted in cassava. With promising future of cassava as an alternative biofuel crop (Nguyen et al., 2007), it would be essential to gain as much information as possible on the processes that underlie sucrose-to-starch transition and starch biosynthesis. As the first steps toward this goal, we isolated partial cDNA fragments encoding MeSPS and MeSPP and three full-length clones of Me14-3-3, namely Me14-3-3; 1, Me14-3-3; 2 and Me14-3-3; 3 cDNAs, from cassava. Subsequently, expression analyses by RNA gel blot and semi-quantitative RT-PCR were carried out to compare differential expression patterns of MeSPS, MeSPP and Me14-3-3 in source and sink organs of plants grown in wet and dry seasons. As a result, it was observed that accumulation of MeSPS, MeSPP and Me14-3-3 transcripts was affected to a certain degree by fluctuating level of rainfall. For cassava, changes in the apparent levels of MeSPS, MeSPP and Me14-3-3 transcripts are probably considered essential for both growth and survival since they allow plants to respond to the cellular demand that might change according to the season.

\section{Materials and Methods}

\subsection{Plant Materials}

Cassava cultivar Kasetsart 50 (KU50) was grown in a field at Rayong Field Crops Research Center, Thailand in two different seasons. The dry crop was planted after the rainy season (in October), while the wet crop was planted during the rainy season (in May) of the following year. After 3, 6, 9 and 12 months of plantation, three plants were harvested and three storage roots were collected from each plant, washed, cut into small pieces and stored at $-80^{\circ} \mathrm{C}$. In addition to storage roots, leaves at two different developmental stages were collected, both during the day and at night. Young leaves were defined as the first unfolded leaves located at the top of each plant, while mature leaves were fully expanded with no visible sign of initial senescence.

\subsection{RT-PCR Amplification of SPS, SPP and 14-3-3 cDNA Fragments}

Total RNA $(1 \mu \mathrm{g})$ extracted from frozen cassava tissues was used as template in first strand cDNA synthesis reactions and then in PCR to obtain partial cDNA fragments, namely MepSPS, MepSPP and Mep14-3-3. The primers used in PCR reactions were designed from consensus sequences of plant SPSs [SPS/1, CATGTG(CT)TGG(CA)G(GA)AT(TC)TGG; SPS/2, GCACCATCAACAAA(TC)TC(AC)GG], SPPs [SPP/1, CTCTTG(C/T)(C/T)TGTGG(T/C)GACTC; SPP/2， T(T/G)(C/T)TTCCC(T/A/C)T(G/T)TTTGTC(T/A)CCA ] and $14-3-3 \mathrm{~s} \quad[14-3-3 / 1, \quad \mathrm{C}(\mathrm{C} / \mathrm{G}) \mathrm{ATTGA}(\mathrm{G} / \mathrm{A}) \mathrm{CAGAAGGA}(\mathrm{G} / \mathrm{A}) \mathrm{GA} ; \quad 14-3-3 / 2, \quad$ TCAGG(A/T/C)GAGTT $(\mathrm{C} / \mathrm{G} / \mathrm{A}) \mathrm{A}(\mathrm{A} / \mathrm{G})(\mathrm{A} / \mathrm{G}) \mathrm{ATCTC}]$.

\subsection{Construction and Screening of cDNA Libraries}

Leaf and storage root cDNA libraries were constructed by inserting poly $(\mathrm{A})^{+}$RNA into $\lambda$ TriplEx 2 vector arms (SMART cDNA library construction kit, ClonTech). Hybridization was carried out at $65^{\circ} \mathrm{C}$ for $16-18 \mathrm{hrs}$ in the presence of $\left[{ }^{32} \mathrm{P}\right]$-labeled Mep14-3-3 DNA probe followed with washing under high stringency conditions (Church \& Gilbert, 1984). By utilizing the cre-lox excision sites in $\lambda$ TriplEx2, pTriplEx2 vector carrying a cassava cDNA insert was obtained and sequenced.

\subsection{Genomic Southern Blot}

Aliquots of cassava genomic DNA $(15 \mu \mathrm{g})$ were digested with restriction endonucleases (BamHI, ClaI, EcoRI, EcoRV, HindIII, KpnI, MluI, SacI, SalI and XbaI), fractionated on $0.8 \%$ agarose gels and blotted onto Hybond-N ${ }^{+}$(GE Healthcare Life Sciences). The blot was hybridized with MepSPS, MepSPP and Me14-3-3;1 
cDNAs, labeled with digoxigenin (DIG)-dUTP (Roche). Hybridization was carried out at $68^{\circ} \mathrm{C}$ followed with washing at $65^{\circ} \mathrm{C}$ (Engler-Blum et al., 1993). After stripping, re-hybridization under lower stringency conditions (hybridization at $60^{\circ} \mathrm{C}$ followed with washing at $58^{\circ} \mathrm{C}$ ) was performed.

\subsection{Expression Analysis by RNA Gel Blot and Semi-quantitative RT-PCR}

RNA gel blot analysis was carried out using $30 \mu \mathrm{g}$ of total RNA extracted from leaf and storage root tissues of cassava. Hybridization was carried out for $16 \mathrm{hr}$ at $65^{\circ} \mathrm{C}$ in the presence of MepSPS, MepSPP or Me14-3-3;1 DNA probes, labeled with $\left[{ }^{32} \mathrm{P}\right]-\mathrm{dCTP}$, followed with washing under high stringency conditions. Equal loading of RNA from various samples was determined by staining the membrane with methylene blue solution $(0.04 \%$ methylene blue in $0.5 \mathrm{M}$ sodium acetate; $\mathrm{pH}$ 5.2).

To perform semi-quantitative RT-PCR, $1 \mu 1$ of cDNA template generated from 3- $\mu \mathrm{g}$ DNase-treated RNA samples was used as template in a 50- $\mu \mathrm{l}$ PCR reaction containing $0.4 \mu \mathrm{M}$ of the gene-specific primers, $200 \mu \mathrm{M}$ dNTPs and 1 unit Taq DNA polymerase (Fermentas). The gene-specific primers were SPS/3 (CATGTGTTGGAGGATT TGG), SPS/4 (GCACCATCAACAAATTCCG), SPP/3 (CTCTTGTTTGTGTGGTGACTCTG), SPP/4 (TTCTT CCCTTTTTTGTCTCCA), 14-3-3/3 (ATTGAGCAGAAGGAAGAG), 14-3-3/4 (ACGGTCAGGTGAGTTTAG), Actin/1 (CATGAGACTACATACAACTCCATC) and Actin/2 (TCGTACTCAGCCTTGGCAATCCAC). Subsequently, PCR was carried out using the conditions that had previously been optimized to allow the detection of MeSPS, MeSPP and Mel4-3-3 transcripts within the linear amplification phase $\left(5 \mathrm{~min}\right.$ at $95^{\circ} \mathrm{C}, 32$ cycles of $1 \mathrm{~min}$ at $94^{\circ} \mathrm{C}, 1 \mathrm{~min}$ at $52^{\circ} \mathrm{C}$ (for MeSPS and MeSPP) or $48^{\circ} \mathrm{C}$ (for Me14-3-3 and MeActin) and 2 min at $72^{\circ} \mathrm{C}$, followed by $10 \mathrm{~min}$ at $72^{\circ} \mathrm{C}$ ). The signals obtained from RNA gel blot and semi-quantitative RT-PCR were analyzed using Quantity One Software available in the Molecular Imager GS-800 calibrated densitometer (Bio-Rad).

\section{Results}

\subsection{Cloning of cDNAs Encoding 14-3-3 Proteins in Cassava}

The Arabidopsis genome contains up to 15 genes, 12 of which have been found to express and thus encode different GF14 isoforms. These GF14s contain a highly conserved core that is flanked by divergent termini. Degenerate primers designed from the core consensus sequences of Arabidopsis GF14s were used in RT-PCR to obtain a partial 14-3-3 cDNA from cassava. In the presence of leaf RNA templates, an amplified fragment of 373 bp, which showed significantly high degree of similarity to 14-3-3 mRNAs from plants, was obtained. To indicate that the resulting 373-bp cDNA encodes a partial 14-3-3 cDNA in cassava, the term Mep14-3-3 cDNA was given and used throughout this study.

To gain information on the genes encoding 14-3-3 proteins in cassava, leaf and storage root cDNA libraries of cassava cv. KU50 were screened using Mep 14-3-3 cDNA, labeled with [ $\left.{ }^{32} \mathrm{P}\right]$-dCTP, as probe. Screening of $1.6 \mathrm{x}$ $10^{5}$ plaque-forming units of the leaf cDNA library yielded four positive clones, which differ from each other at the length of $5^{\prime}$ region. Therefore, only the clone that carries the longest cDNA insert, Me14-3-3;1, was further characterized (GenBank ID: DQ013887). By screening the storage root cDNA library, two full-length clones encoding 14-3-3 proteins, Me14-3-3;2 and Me14-3-3;3, were identified (GenBank IDs: GQ922216 and GQ922217 respectively).

Me14-3-3;1, Me14-3-3;2 and Me14-3-3;3 cDNA are 1,186, 1,463 and 1,122 bp long, respectively (Figure 1). Although the length of these cDNAs is different, their coding regions are similarly composed of 795 nucleotides, thus coding for a polypeptide of 264 amino acids or $30 \mathrm{kDa}$. However, Me14-3-3;1 cDNA shared higher degree of similarity to $\mathrm{Me} 14-3-3 ; 3$ cDNA (98\% identity at nucleotide level and $95 \%$ identity at amino acid level) than to Me14-3-3;2 cDNA (78\% and 100\% identity at nucleotide and amino acid level respectively). When compared with $\operatorname{grf}$ genes from Arabidopsis, the cassava cDNAs showed highest degree of similarity $(84-90 \%$ identity) to grf3 (GenBank ID: L09110), grf7 (GenBank ID: U60445) and grf5 (GenBank ID: L09109), respectively. To gain information on Me14-3-3 encoding genes that might belong to other subgroups in the Arabidopsis phylogenetic tree, Me14-3-3;1, Me14-3-3;2 or Me14-3-3;3 were used to BLAST against the recently released cassava genome sequence database (http://www.phytozome.net/cassava). As a result, we identified 14 regions on the cassava genome that most likely contain Me14-3-3-encoding genes. Genes within these regions could give rise to major mRNA species of 16 different types, one of which possibly represents another Me14-3-3 cDNA isolated from storage roots of cassava cv. KU50 (Sojikul et al., 2010). As expected, the polypeptides derived from these 16 mRNA species appeared to show high degree of similarity to those encoded by the Arabidopsis $g r f$ genes (Figure 2). 


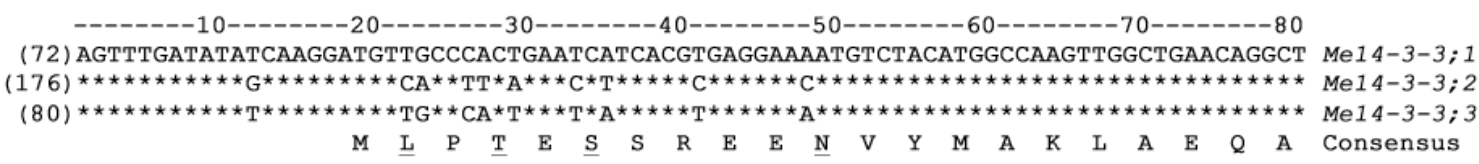

(152) GAACGTTATGAGGAAATGGTGGATTTATGGAAAAGGTGCAAAGACAGTGGATGTGGAGGAGCTAACTGTGGAGGAAAG Me14-3-3; 1

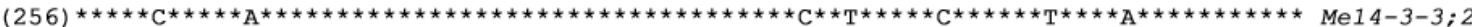

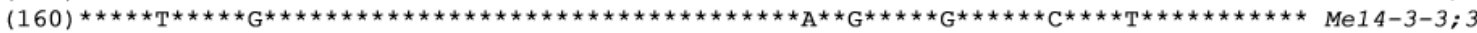
$\begin{array}{llllllllllllllllllllllllllllllllll}E & R & Y & E & E & M & V & E & F & M & E & K & V & A & K & T & V & D & V & E & E & L & T & V & E & E & R & C o n s e n s u s\end{array}$

(232) GAATCTTCTCTCTGTCGCTTACAAGAACGTCATTGGGGCTAGAAGGGCTTCATGGAGGATAATCTCTTCCATTGAGCAGA Me1 4 -3-3; 1

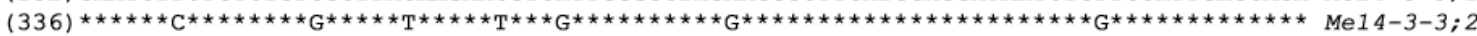

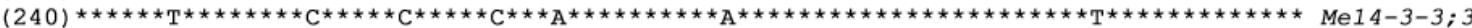
$\begin{array}{llllllllllllllllllllllllllllllllll}N & L & L & S & V & A & Y & K & N & V & I & G & A & R & R & A & S & W & R & I & I & S & S & I & E & Q & C o n s e n s u s\end{array}$

(312) AgGAAGAGAGCAGAGGAAATGAGGATCACGTGTCAATAATTAAGGAGTACAGAGGTAAGATTGAAGCTGAGCTGAGCAAG Me14-3-3; 1

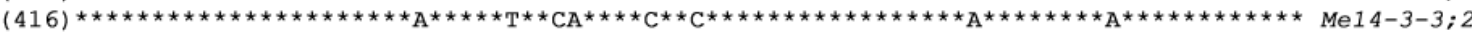

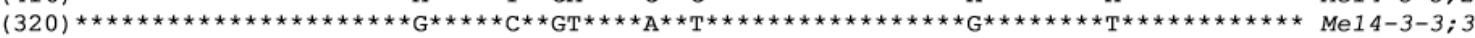
$\begin{array}{lllllllllllllllllllllllllllll}K & E & E & S & R & G & N & E & D & H & V & S & I & I & K & E & Y & R & G & K & I & E & A & E & L & S & K & C o n s e n s u s\end{array}$

(392) ATTTGTGATGGGATCTTGAGCCTCCTTGAGTCGCATCTCATTCCCTCTGCCTCATCTGCTGAGTCTAAGGTATTCTACCT Me14-3-3; 1

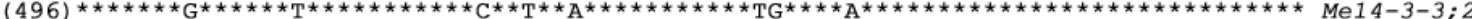

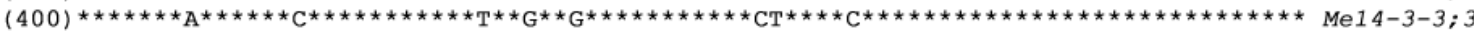
$\begin{array}{llllllllllllllllllllllllllll}I & C & D & G & I & L & S & L & L & \underline{E} & S & H & L & I & P & \underline{S} & A & S & S & A & E & S & K & V & F & Y & L & C o n s e n s u s\end{array}$

(472) CAAGATGAAGGGTATTATCACCGGTATCTTGCGGAGTCAAGACTGCAGCTGAGAGGAAGGAGCTGCTGAGAGTACTT Me14-3-3; 1

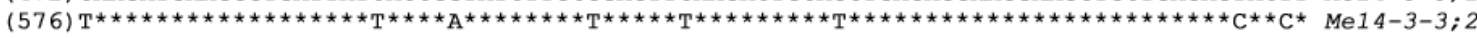

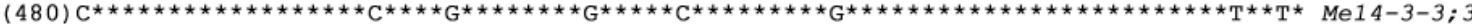
$\begin{array}{llllllllllllllllllllllllllll}K & M & K & G & D & Y & \underline{H} & R & Y & L & A & E & F & K & T & A & \underline{A} & E & R & K & E & A & A & E & S & T & C o n s e n s u s\end{array}$

(552) TGTTGGCATACAAGTCTGCCCAGGATATTGCCCTTGCTGATCTCGCTCCTACCCACCCAATAAGGCTTGGGCTTGCCCTT Me14-3-3; 1

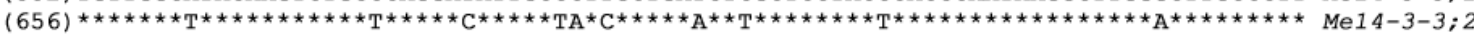

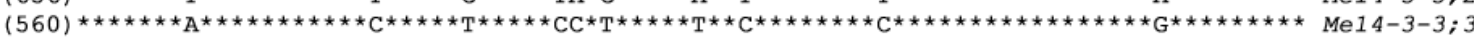

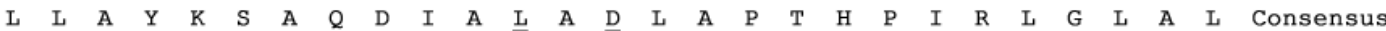

(632) AACTTCTCCGTGTTCTATTATGAGATCCTAAACTCACCTGACCGTGCTTGTAATCTAGCCAAGCAGGCCTTTGATGAGGC Me14-3-3; 1

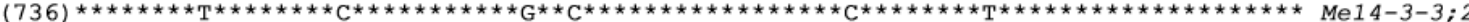

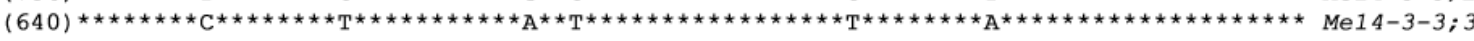
$\begin{array}{llllllllllllllllllllllllllll}N & F & S & V & F & Y & Y & E & I & L & N & S & P & D & R & A & C & N & L & A & K & Q & A & F & D & E & A & C o n s e n s u s\end{array}$

(712) TATTTCTGAGCTGGATACATTGGGTGAGGAATCTTACAAGGATAGTACATTGATCATGCAACTTCTCCGAGACAATCTGA Me14-3-3; 1

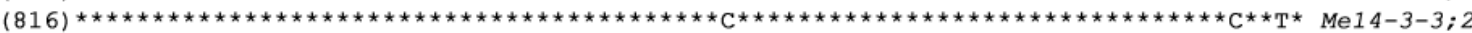

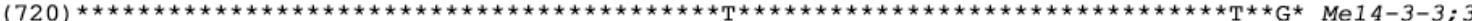
$\begin{array}{lllllllllllllllllllllllllllll} & \mathrm{S} & \mathrm{E} & \mathrm{L} & \mathrm{D} & \mathrm{T} & \mathrm{L} & \mathrm{G} & \mathrm{E} & \mathrm{E} & \mathrm{S} & \mathrm{Y} & \mathrm{K} & \mathrm{D} & \mathrm{S} & \mathrm{T} & \mathrm{L} & \mathrm{I} & \mathrm{M} & \mathrm{Q} & \mathrm{L} & \mathrm{L} & \mathrm{R} & \mathrm{D} & \mathrm{N} & \mathrm{L} & \text { Consensus }\end{array}$

(792) CGCTCTGGACTTCTGATATCACGGACGAAGCTGGGGATGAGATCAAGGATGCATCAAAACGGGAATCAGGCGAGGGACAG Me14-3-3; 1

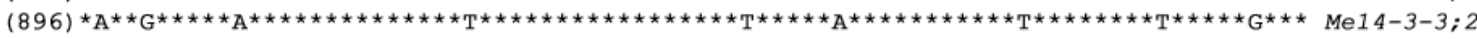

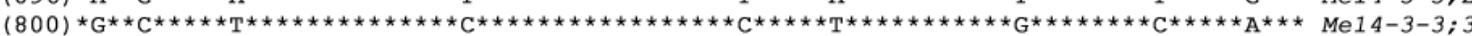

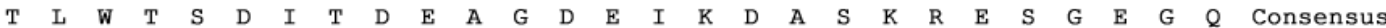

(872) CCGCAACAGTGATGAGTTAA-ATTCGTAGGA--CGTGTAATGTGTACTTCTA-TATCTTGTGACTTCTGAGTAGATGCCA Me14-3-3; 1

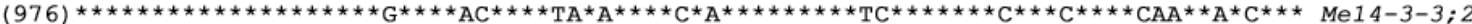

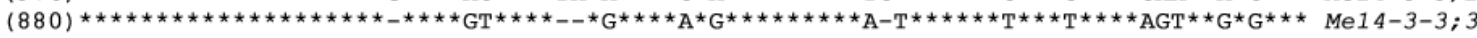
$P \quad Q \quad Q$ Consensus

Figure 1. Alignment of Me14-3-3;1 (GenBank ID: DQ013887), Me14-3-3;2 (GenBank ID: GQ922216) and Me14-3-3;3 (GenBank ID: GQ922217)

Amino acids deduced from these cDNAs were shown; however, at the positions where substitution occurs only the amino acids that appear as majority were indicated and underlined. 


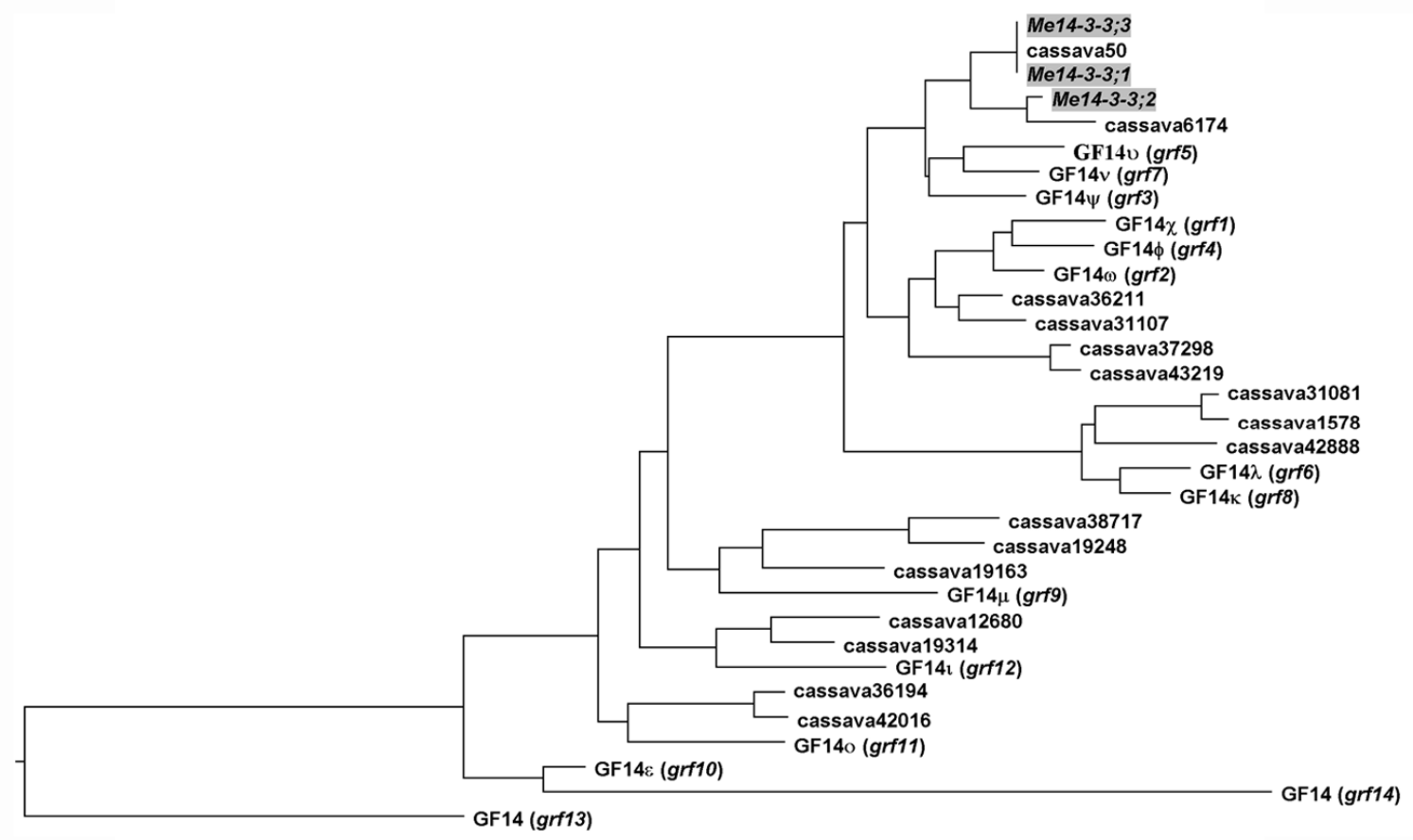

Figure 2. Phylogenetic relationship of 14-3-3 proteins constructed from deduced amino acid sequences of cassava and Arabidopsis

The deduced amino acid sequences are Me14-3-3;1, Me14-3-3;2 and Me14-3-3;3 cDNA (this study) and 16 different mRNA species from cassava obtained from the cassava genome sequence database, and also from 14

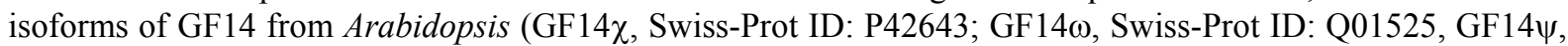

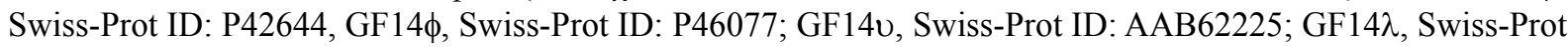
ID: P48349; GF14v, Swiss-Prot ID: AAD51782, GF14к, Swiss-Prot ID: AAD51783; GF14 $\mu$, Swiss-Prot ID: AAD51784; GF14ع, Swiss-Prot ID: P48347; GF14o, Swiss-Prot ID: AAG47840; GF14ı, Swiss-Prot ID: AAF98570. Three remaining isoforms of Arabidopsis GF14, which appear under Swiss-Prot IDs: AAG52105, AAF87262 and AAD28654, are currently designated as 14-3-3 like proteins since expression of the genes that encode them have not yet been observed. The grf gene encoding each of these GF14s are specified in brackets.

\subsection{Cloning of Partial cDNAs Encoding SPS and SPP in Cassava}

Partial cDNAs encoding MeSPS and MeSPP were obtained by RT-PCR using cassava leaf RNA as template. Amplification of MepSPS cDNA was carried out using primers designed from consensus sequences of the SPS $\mathrm{N}$-terminal glycosyltransferase domain. The amino acid sequence deduced from the MepSPS cDNA showed 85\% and $73 \%$ identity to the two isoforms of Arabidopsis SPS family A encoded by AtSPS1F (GenBank ID: NM_122035) and AtSPS2F (GenBank ID: NM_121149), while it shared only $63 \%$ and $54 \%$ identity to the family B (AtSPS3F; GenBank ID: NM_100370) and family C isoforms (AtSPS4F; GenBank IDs: NM_001036532 and NM_117030), respectively. These results therefore suggest that the 621-bp MepSPS cDNA is derived from the gene encoding SPS family A in cassava. In addition to the glycosyltransferase domain, MepSPS cDNA also encodes two serine residues, whose positions correspond to the Ser-158 and Ser-229 of spinach SPS (Figure 3).

To obtain MepSPP cDNA, RT-PCR was carried out using forward and reverse primers derived from consensus sequences within the N-terminal L-2-haloacid dehalogenase (HAD) domain and the C-terminal extension region of plant SPPs, respectively. 


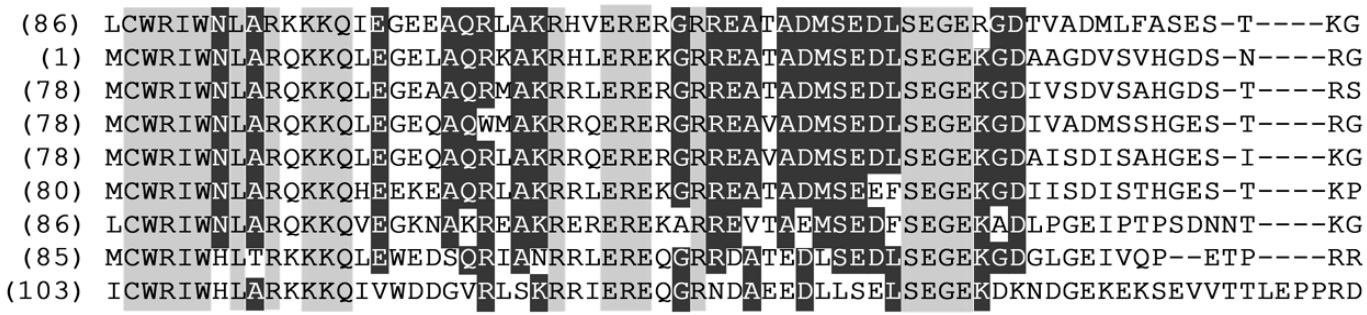

(103) ICWRIWHLARKKKQIVWDDGVRLSKRRIEREQGRNDAEEDLLSELSEGEKDKNDGEKEKSEVVTTLEPPRD

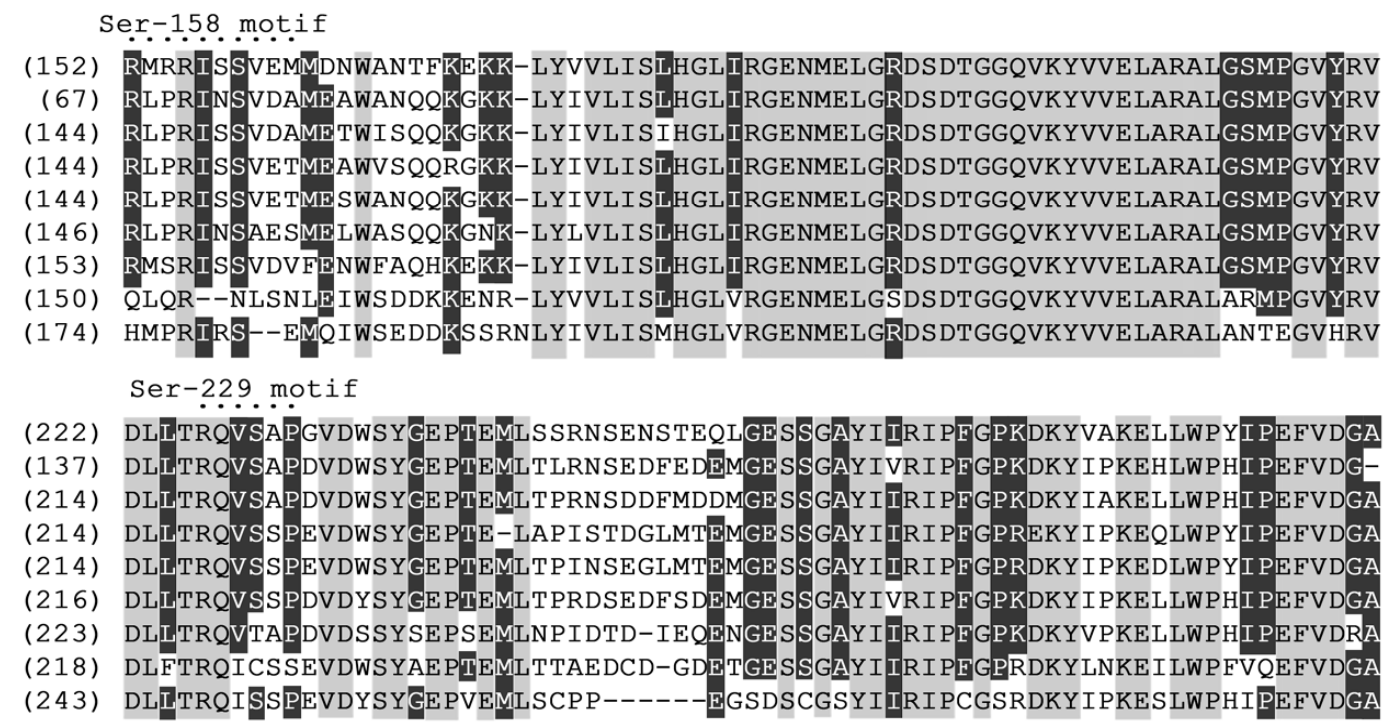

SOSPS

MepSPS

Cusps 1

StSPS

IbSPS

AtSPS1F

AtsPS2F

AtSPS3F

AtsPS $4 \mathrm{~F}$

SoSPS

MepSPS

Cusps 1

StSPS

IbSPS

AtSPS1F

AtSPS2F

AtSPS $3 \mathrm{~F}$

AtSPS $4 \mathrm{~F}$

SoSPS

MepSPS

CuSPS1

StSPS

IbSPS

AtSPS1F

AtSPS2F

AtSPS $3 F$

AtSPS $4 \mathrm{~F}$

Figure 3. Alignment of amino acid sequences encoded by 621-bp of SPS cDNA from cassava, spinach, citrus, potato, sweet potato and Arabidopsis

The amino acid sequences encoded by 621-bp MepSPS cDNA (GenBank ID: GQ922214), SoSPS mRNA from Spinacia oleracea (GenBank ID: L04803), CuSPS1 mRNA from Citrus unshiu (GenBank ID: AB005023), StSPS from Solanum tuberosum (GenBank ID: X73477), IbSPS mRNA from Ipomoea batatas (GenBank ID: AF439861) and Arabidopsis thaliana mRNAs designated as AtSPS1F (GenBank ID: NM_122035), AtSPS2F (GenBank ID: NM_121149), AtSPS3F (GenBank ID: NM_100370) and AtSPS4F mRNAs (GenBank ID: NM_117080). Residues conserved in all and most of the sequences are shown in gray and black, respectively. The residues that are included in the Ser-158 and Ser-229 motifs are indicated by dots above.

\subsection{DNA Gel Blot Analysis of Genes Encoding MeSPS, MeSPP and Me14-3-3}

Based on the allopolyploid nature of cassava, the number of bands, observed by DNA gel blot, is not necessarily equal copy number of genes present in the genome. In our case, interpretation of the results from DNA gel blot analysis of genes encoding MeSPS, MeSPP and Me14-3-3 is even more complicated due to insufficient information on the genomic sequences. As a result, multiple banding patterns observed by DNA gel blot might derive from different regions of the same gene. To minimize this problem, restriction maps of genes from various plant species were determined and only the enzymes that cannot cut within the genes were chosen for this study. When using MepSPS cDNA as probe, multiple digested products could be observed (Figure 4a). The result has therefore suggested that multiple copies of MeSPS gene are present in the cassava genome. Based on the multiple banding patterns detected by the MepSPP cDNA probe (Figure 4b), MeSPP should also exist as multiple copy genes in the cassava genome.

Unlike MeSPS and MeSPP, DNA gel blot analysis using Me14-3-3;1 cDNA probe could result in the detection of only a few fragments (Figure 4c). To determine that the genes of significantly lower similarity might exist in the cassava genome, the probe was striped and hybridization at low stringency condition was performed. According to Figure 4d, several additional fragments were observed, thus indicating that multiple copies of Me14-3-3 genes exist in the cassava genome. 

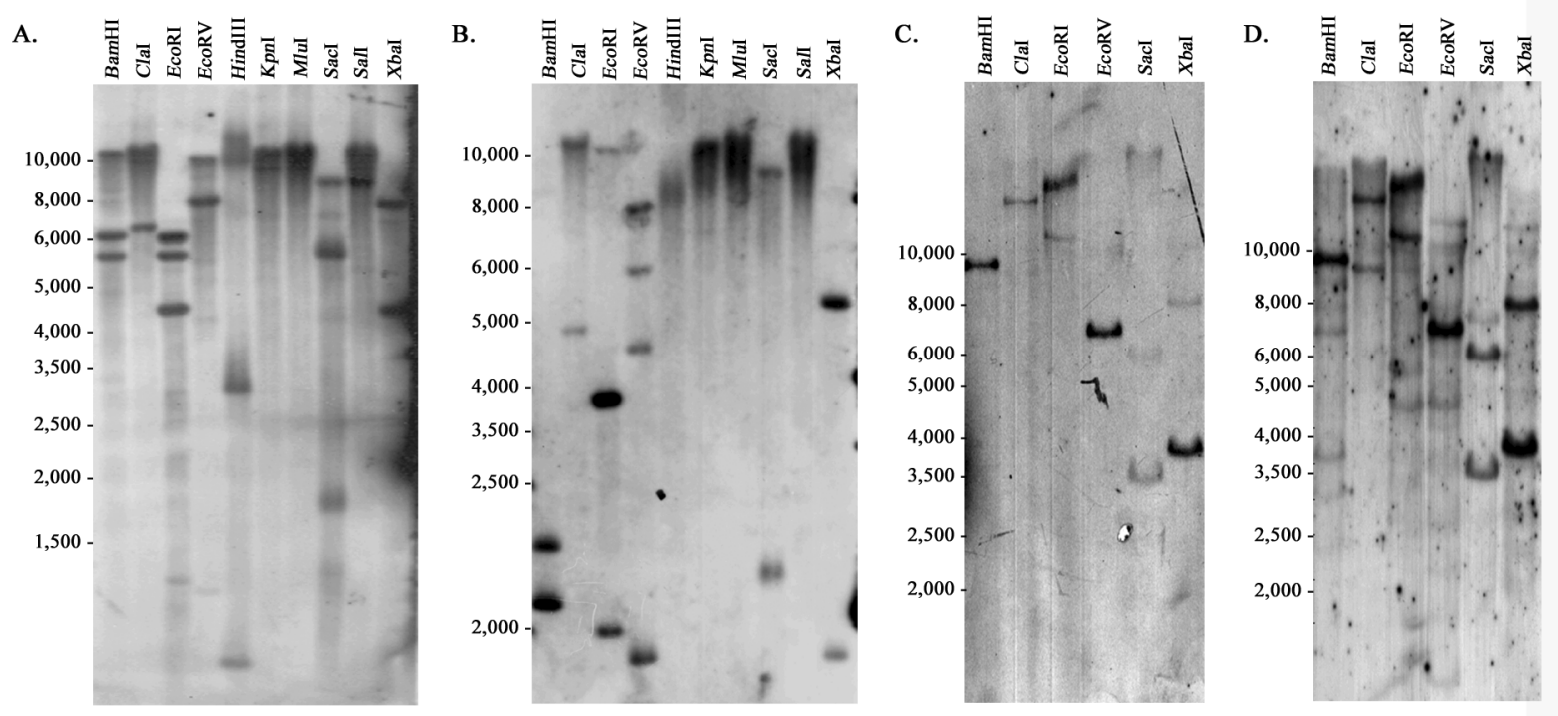

Figure 4. DNA gel blot analysis probed with MepSPS (A), MepSPP (B) and Me14-3-3;1 (C and D carried out under high and low stringency conditions, respectively)

The sizes in base pairs of the DNA molecular weight bands are indicated on the left.

\subsection{Differential Expression Patterns of MeSPS, MeSPP and Me14-3-3}

RNA gel blot was employed to investigate temporal expression patterns of MeSPS, MeSPP and Me14-3-3 in storage roots of cassava planted in wet and dry season. According to Figure 5, different sized transcripts of 3.5 and $2.0 \mathrm{~kb}$ were detected by MepSPS cDNA probe. Based on the size of other plant SPS mRNAs, the 3.5-kb transcript would most likely represent full-length MeSPS mRNA, while the presence of smaller sized transcript has not been observed elsewhere. Therefore, it remains to be investigated whether the 2.0-kb MeSPS mRNA arises from partial degradation of the large transcript, from alternative splicing of the single pre-mRNA, or, alternatively, from different genes. No matter what would be the cause for the presence of two different sized MeSPS transcripts, the results shown in Figure 4 indicated that both transcripts had comparatively similar expression patterns and their levels in cassava storage roots also followed each other closely. However, we observed that the signals obtained from RNA gel blot were considered fairly low even from $30 \mu \mathrm{g}$ of total RNA as starting material. Alternatively, semi-quantitative RT-PCR was carried out using the conditions (i.e. cycle number and concentrations of primers, template and $\mathrm{MgCl}_{2}$ ) that result in the detection of MeSPS, and also $M e S P P$ and Me14-3-3, in the linear amplification phase. Along with cDNAs, RNA samples pre-treated with DNaseI were also used as templates in RT-PCR. This was to ensure that the RNA samples used in first strand cDNA synthesis reactions were devoid of genomic DNA contamination.

Results obtained from RNA gel blot (Figure 5) and semi-quantitative RT-PCR (Figure 6) similarly indicated that the levels of MeSPS transcripts increased as the roots of dry crop developed from 3 to 6 and 9 months old. As the plants matured further, the levels of MeSPS mRNAs decreased gradually. For plants grown in the wet season, abundant levels of MeSPS transcripts were present in storage roots at 3 months old and then decreased to significantly low levels at later stages.

Unlike MeSPS, the levels of MeSPP transcript fluctuated only slightly during the course of storage root development (Figure 5). When growing season was taken into account, we observed that MeSPP transcript was present at slightly higher level in plants grown in the wet season (Figures 5 and 6). Although this finding was observed by both RNA gel blot and semi-quantitative RT-PCR, the high sensitivity of PCR allowed the result to be more easily detected. 


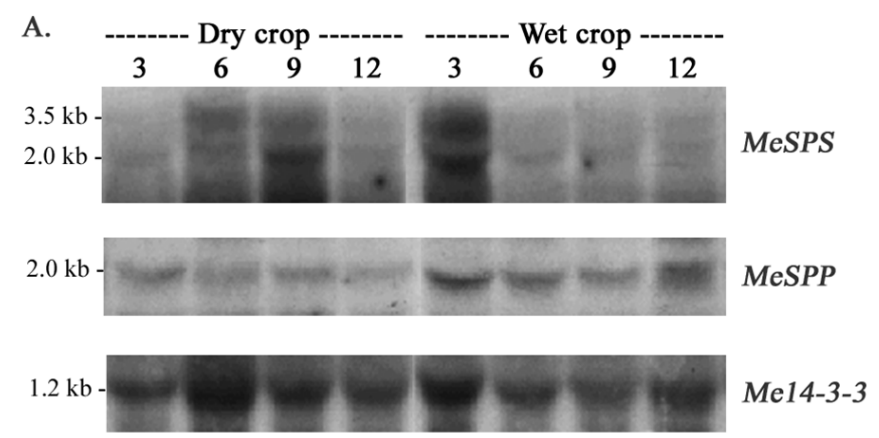

B.

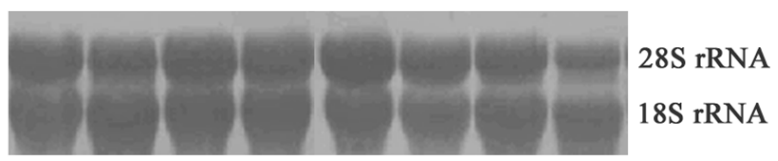

Figure 5. Temporal expression patterns of MeSPS, MeSPP and Me14-3-3 in storage roots of cassava

The cassava plants were grown for 3, 6, 9 and 12 months in wet and dry season. RNA gel blot analysis was carried out by priming $30 \mu \mathrm{g}$ of total RNA with MeSPS, MeSPP or Me14-3-3;1, labeled with [ $\left.{ }^{32} \mathrm{P}\right]-\mathrm{dCTP}$, as probes (A). Equal loading of RNA from various samples was determined by staining the membrane with methylene blue solution (B).

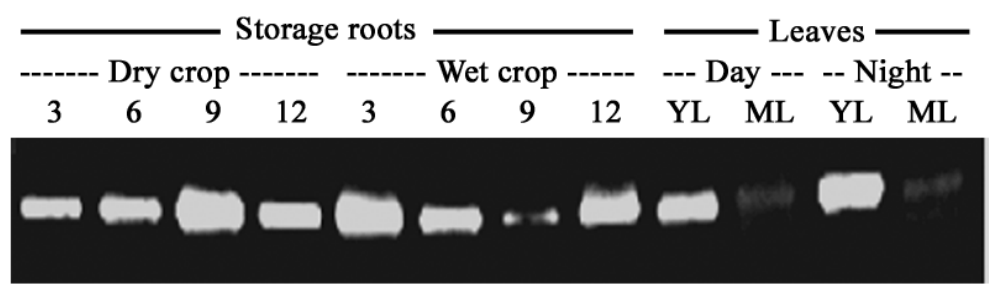

\section{MeSPS}

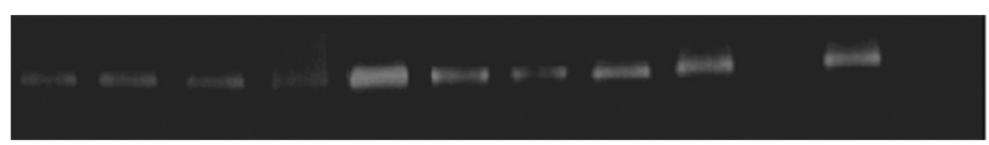

$\operatorname{MeSPP}$

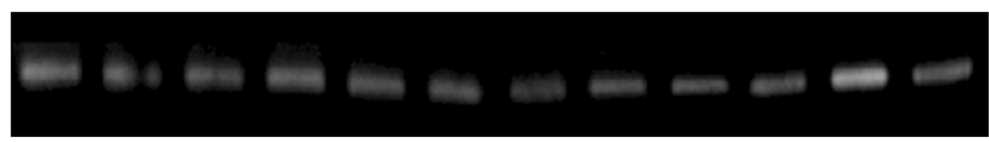

Me14-3-3

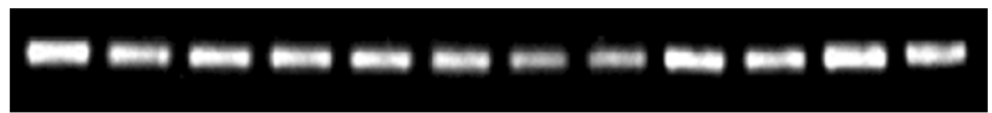

Actin

Figure 6. Expression profiles of MeSPS, MeSPP and Me14-3-3 in storage roots of cassava

The cassava plants were grown for 3, 6, 9 and 12 months in wet and dry season and in young (YL) and mature (ML) leaves collected during the day or at night observed by semi-quantitative RT-PCR using actin as an internal control

Expression analysis of Me14-3-3 by RNA gel blot resulted in the detection of 1.2-kb transcript in storage roots of both wet and dry crops (Figure 5). Although the levels of 1.2-kb transcript seemed to rarely fluctuate, it was nonetheless possible to indicate that accumulation of Me14-3-3 transcript was present at slightly higher level in dry crop roots at 6 months old and in wet crop roots at 3 months old. Based on the size of Me14-3-3 cDNAs (Figure 1), slight variation in 1.2-kb transcript level could only reflect the accumulation of $M e 14-3-3 ; 1$ and Me14-3-3;3 transcripts in storage root tissue of cassava. Unlike results obtained from RNA gel blot, semi-quantitative RT-PCR indicated that Me14-3-3 was expressed at relatively stable levels in all cassava tissues 
tested in this study (Figure 6). Since the primers used in semi-quantitative RT-PCR were designed from the core consensus sequence of Me14-3-3 cDNAs, the results obtained did not allow us to identify which of the Me14-3-3 encoding genes that might be differentially expressed during the course of cassava root development. Given that this is the first report on Me14-3-3s from cassava and the three Me14-3-3 mRNAs showed extremely high degree of similarity to each other (78-98\% identity at nucleotide level and $98-100 \%$ identity at amino acid level), it would be very difficult to obtain primers that are specific to each Me14-3-3 encoding gene. Based on the results obtained, however, it is possible to indicate that temporal expression patterns of Me14-3-3, particularly those derived from the genes encoding Me14-3-3;1 and Me14-3-3;3, vary depending on the root developmental stage and different planting season.

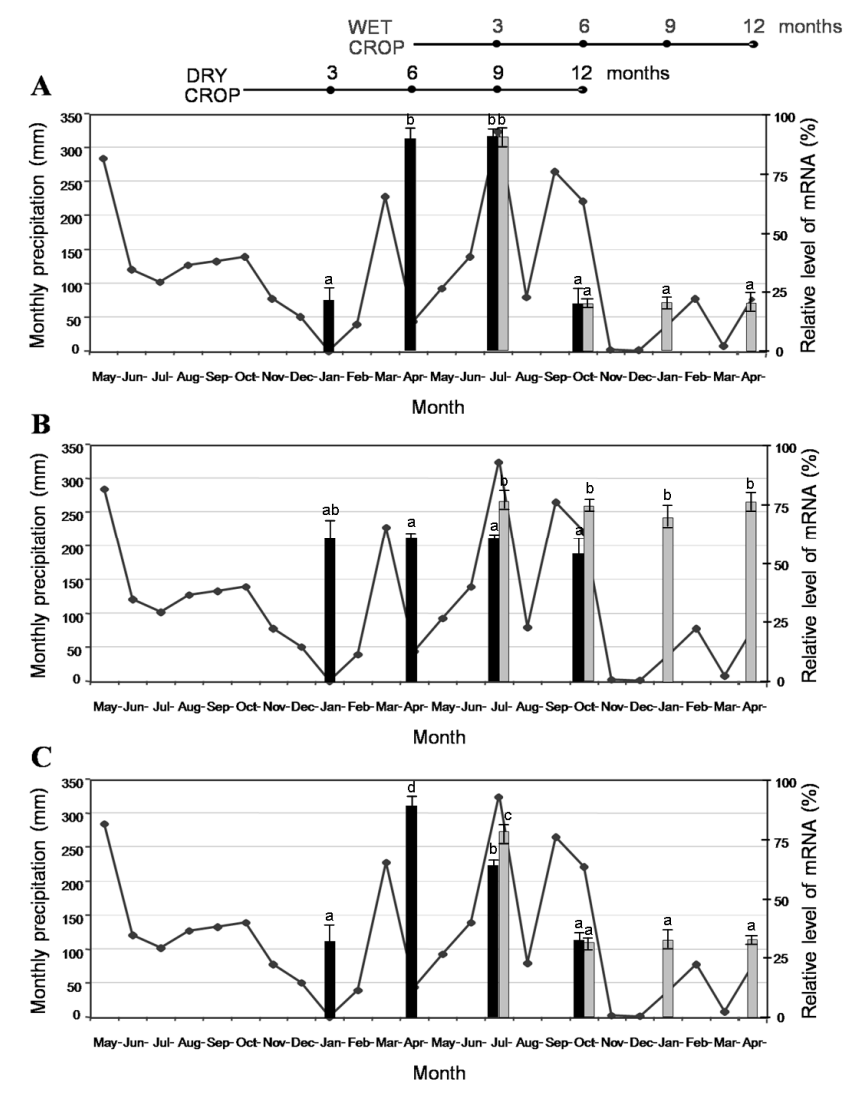

Figure 7. Accumulation of $\operatorname{MeSPS}(\mathrm{A}), \operatorname{MeSPP}(\mathrm{B})$ and Me14-3-3 (C) gene transcripts in response to fluctuating levels of rainfall, dry crop (black) and wet crop (grey)

RNA gel blot analysis was carried out using $30 \mu \mathrm{g}$ of total RNA extracted from 3 storage root tissues obtained from 1 cassava plant. The 3 independent replicate experiments were performed. Intensity of the signals observed by RNA gel blot analyses was analyzed by QuantityOne software (Bio-rad) and plotted vs. monthly precipitation levels. Data correspond to the mean of 3 independent replicate experiments $(n=3)$, the error bars represents the standard error of the mean (SE). The significant differences were determined by DNMRT using the SPSS software package. Values followed by the same letter are not significantly different $(p<0.01)$ using DNMRT.

To obtain information on MeSPS, MeSPP and Me14-3-3 expression patterns in source tissues, cassava leaves at different developmental stages (young and mature) and carbon partitioning status (harvested during the day and at night) were utilized as starting materials for semi-quantitative RT-PCR analysis (Figure 6). In young leaves, abundant level of MeSPS transcript was observed and its level seemed to be slightly higher in leaves harvested at night. In mature leaves, a significantly low level of MeSPS mRNA was observed and its levels did not seem to be affected by the day/night cycle. For MeSPP, accumulation of the transcript was observed only in young leaves and its levels in leaves harvested at different time of day were comparatively stable. Similar to the results observed in storage root tissue, the signals derived from Me14-3-3 transcripts appeared to be unchanged (Figure 
6). Again, this possibly resulted from the inability of the primers to distinguish between each of the Me14-3-3 cDNA template. Accordingly, stable accumulation of the 375-bp RT-PCR product would reflect ubiquitous existence of 14-3-3 proteins in cassava tissues rather to indicate that all the 14-3-3-encoding genes are stably expressed in all types of cassava tissues. Accumulation of MeSPS, MeSPP and Me14-3-3 transcripts in response to fluctuating levels of rainfall, dry crop and wet crop were determined (Figure 7). And the cis-acting elements in the 5'-upstream region of MeSPS, MeSPP and Me14-3-3 genes were also analyzed (Figure 8).

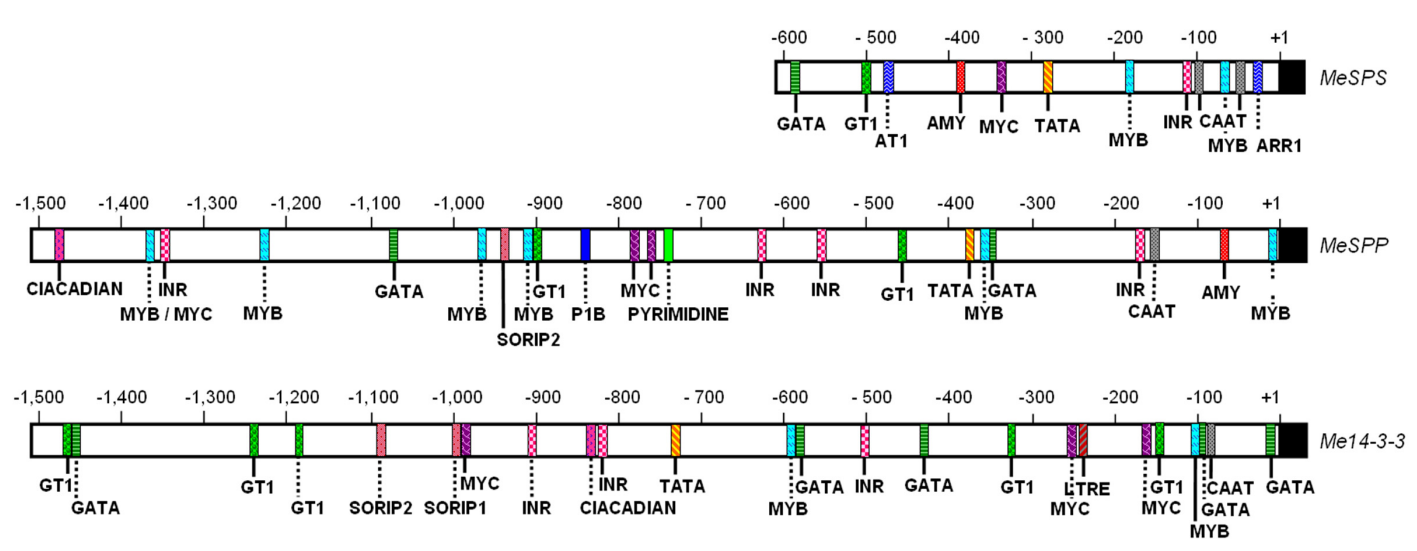

Figure 8. Diagrammatic illustration of various cis-acting elements in the $5^{\prime}$-upstream region of MeSPS, MeSPP and Me14-3-3 genes

Cis-acting elements in the $5^{\prime}$-upstream region were analyzed by PLACE software (http://www.dna.affrc.go.jp/PLACE/). Positions of the cis-acting elements, including dehydration responsive elements (i.e. Myb and Myc binding sites) and a drought responsive LTRE box, are indicated with negative numbers in relation to the translational start site at +1 position.

\section{Discussion}

The 14-3-3s are ubiquitous proteins that have been observed in virtually every eukaryotic organism. According to Rosenquist et al. (2001), the Arabidopsis genome contains up to 15 genes ( $g r f l$ to grf15), 12 of which have been found to express and thus encode different GF14 isoforms designated as chi $(\chi)$, omega $(\omega)$, psi $(\psi)$, phi $(\phi)$, upsilon ( $v)$, lambda $(\lambda)$, nu (v), kappa $(\kappa)$, mu ( $\mu$ ), epsilon ( $(\varepsilon)$, omicron (o) and iota ( 1$)$. These GF14s contain a highly conserved core that is flanked by divergent termini. In addition to Arabidopsis, other plants also possess multiple isoforms of 14-3-3 protein, each of which is encoded by highly conserved yet distinct gene (Konagaya et al., 2004; Wu et al., 1997; Xu et al., 2006). In this study, three full-length cDNAs encoding 14-3-3 proteins were isolated from cDNA libraries of cassava. The Me14-3-3;1 cDNA, which was isolated from the leaf cDNA library, is 1,186 bp long, while the Me14-3-3;2 and Me14-3-3;3 cDNAs obtained from the storage root cDNA library were 1,463 and 1,122 bp in length, respectively. Although the length of these cDNA clones is different, they similarly encode a polypeptide of 264 amino acids or $30 \mathrm{kDa}$. Both nucleotide and deduced amino acid sequences of Me14-3-3;1 cDNA are more closely related to those of Me14-3-3;3 than to Me14-3-3;2 cDNA. According to Rosenquist et al. (2001), the Arabidopsis grfl to grfl4 genes have been divided into two major branches in the phylogenetic tree. The first branch is composed of three subgroups; grfl/grf2/grf4, grf3/grf5/grf7 and $g r f 6 / g r f 8$. The other branch includes the remaining grf9 to grfl4. Me14-3-3 cDNAs showed highest degree of similarity $(85-90 \%)$ to members in the grf3/grf5/grf7 subgroup, and then to grfl/grf2/grf4 $(79-83 \%)$ and grf6/grf8 (72-74\%), respectively. According to Sehnke et al. (2000), the products of $g r f 7$ and $g r f 5$ are among two of the four GF14s that were identified to be located inside chloroplast stroma, thus suggesting their specialized role in metabolic pathways of chloroplasts.

When compared with 17 isoforms of tobacco 14-3-3 proteins (Konagaya et al., 2004), the proteins encoded by Me14-3-3 cDNAs showed highest similarity to the tobacco isoform e1 and e2. These tobacco isoforms have been shown to have intermediate binding affinity with SPS (Bornke, 2005). This corresponds to the results observed in spinach (Spinacia oleracea), where direct binding of 14-3-3 to the phosphorylated Ser-229 of SoSPS has been indicated (Toroser et al., 1998). By conducting RT-PCR using degenerate primers designed from consensus 
sequences of plant SPSs, a 621-bp MepSPS cDNA was obtained. Deduced amino acid sequence of the MepSPS cDNA indicate that it encodes a serine residue that corresponds to the Ser-229 of SoSPS. Similar to SPSs from various plant species, the 'Ser-229' of MepSPS is also located within the consensus motif R-X-X-S*-X-P (where $\mathrm{S}^{*}$ indicates the phosphorylated serine). The presence of this motif in MeSPS probably suggested that interaction between MeSPS and Me14-3-3(s) was also conserved in cassava.

Partial cDNAs encoding MeSPS and MeSPP were obtained by RT-PCR using cassava leaf RNA as template. Amplification of MepSPS cDNA was carried out using primers designed from consensus sequences of the SPS $\mathrm{N}$-terminal glycosyltransferase domain. According to BLAST (Mauchler-Bauer et al., 2009), the glycosyltransferase domain encoded by 621-bp MepSPS (GenBank ID: GQ922214) could be further classified as GT1 family, which is exclusively observed in enzymes from plants or photosynthetic bacteria. Phylogenetic analysis of plant SPSs has classified the enzymes into three families, designated as A, B and C, with monocot/dicot diversions present in each family (Langenkamper et al., 2002; Lutfiyya et al., 2007). The amino acid sequence deduced from the MepSPS cDNA showed $85 \%$ and $73 \%$ identity to the two isoforms of Arabidopsis SPS family A encoded by AtSPS1F (GenBank ID: NM_122035) and AtSPS2F (GenBank ID: NM_121149), while it shared only 63\% and $54 \%$ identity to the family B (AtSPS3F; GenBank ID: NM_100370) and family C isoforms (AtSPS4F; GenBank IDs: NM_001036532 and NM_117030), respectively. These results therefore suggest that the 621-bp MepSPS cDNA is derived from the gene encoding SPS family A in cassava.

The glycosyltransferase domain, MepSPS cDNA is found to encode two serine residues, whose positions correspond to the Ser-158 and Ser-229 of spinach SPS. Phosphorylation of the spinach Ser-158 and Ser-229 has been indicated to involve in light/dark modulation and 14-3-3 protein binding, respectively (Huber \& Huber, 1992; Toroser et al., 1998). Similar to the Ser-158 and Ser-229 of spinach leaf SPS, the corresponding residues of cassava SPS were observed within the consensus motif B-Hy-X-B-X-X-S*-X-X-Hy (where B is a basic residue, Hy a hydrophobic residue, $S *$ the phosphorylated serine and $X$ any amino acid) (Huang \& Huber, 2001), and R-X-X-S*-X-P, respectively (Winter \& Huber, 2000).

The MepSPP cDNA was obtained using primers derived from consensus sequences within the HAD domain and the C-terminal extension region of plant SPPs respectively. It is important to note that in addition to the glycosyltransferase domain, plant SPSs also contain an SPP-like domain, which shows some similarity (35\%) to the catalytic HAD domain of SPP (Lunn et al., 2000). By including the fragment that codes for the C-terminal extension region of SPP into the RT-PCR product, non-specific amplification of MeSPS cDNA could therefore be minimized. As expected, the 460-bp RT-PCR product (GenBank ID: GQ922215) not only showed high degree of similarity to SPP mRNAs but encodes a part of the catalytic HAD phosphatase domain containing a part of the third, from a total of three, highly conserved motifs typical of enzymes in the HAD superfamily (Aravind et al., 1998).

When growing season was taken into account, it could be observed that the dry crop was planted when the rainfall level was low and this condition continued until the first onset of rain in the $5^{\text {th }}$ month. At the onset of rain in the $6^{\text {th }}$ month, growth resumes using the energy derived from degradation of reserve starch (Pardales \& Esquibel, 1996; Sriroth et al., 2001). At this stage, the net flux of sucrose metabolism would be toward the rate of sucrose (re)synthesis, thus explaining the increase in MeSPS transcript in the roots of dry crop harvested at 6 months old. Since the conditions that resemble drought followed with the onset of rain still persisted from $6^{\text {th }}$ to $9^{\text {th }}$ month, high level of MeSPS expression could still be observed at 9 months old. With the return of drought in the following year, marked decrease in MeSPS transcript level was observed. This finding was similarly observed in both wet and dry crop, thus indicating that plants grown in different season responded to drought in a similar manner. In growing potato tubers, short-term water deficit was found to activate $S P S$, thus leading to a switch from net sucrose degradation to net sucrose synthesis (Geigenberger et al., 1997 \& 1999). Although our study in cassava produced conflicting results, these studies similarly indicated that differential expression of SPS was essential in water stress response in plants. However, the mechanisms underlying drought-stress responses vary depending on plant species. The change in rainfall level seemed to affect Mel4-3-3 expression in the same way as it did to MeSPS. The relatively similar patterns of MeSPS and Me14-3-3 might indicate that these proteins need to co-exist to form a multi-protein complex. However, it is important to note that the results most likely reflected only the levels of Me14-3-3;1 and Me14-3-3;3 mRNA but not Me14-3-3;2 transcript.

To support the idea that expression of MeSPS, MeSPP and Me14-3-3 could be triggered by seasonal drought stress, $5^{\prime}$-upstream regions of these putative genes were downloaded from cassava genomic sequences in Phytozome database (https://www.phytozome.net/cassava) and analyzed for putative cis-acting elements using PLACE software (http://www.dna.affrc.go.jp/PLACE/) (Higo et al., 1999). As a result, several dehydration responsive elements such as Myb and Myc binding sites as well as a drought-responsive LTRE box (Baker et al., 
1994) were observed. These results therefore suggest that expression of MeSPS and Me14-3-3 genes are influenced by changes in environmental conditions such as drought. In addition, both MeSPS and MeSPP promoters also contain an alpha-amylase (Amy) responsive element (Huang et al., 1990).

By conducting RNA gel blot analysis, we observed two different sized MeSPS transcripts, 3.5 and $2.0 \mathrm{~kb}$ in length, in storage root tissue of cassava. However, the results clearly indicated that both transcripts had comparatively similar expression patterns and their levels in cassava storage roots also followed each other closely. Similarly, multiple SPS transcripts were observed in wheat (Castleden et al., 2004) and banana (do Nascimento et al., 1997). However, identity of the truncated transcripts is still poorly understood. For cassava, it remains to be investigated whether the truncated MeSPS mRNA arises from partial degradation of the $3.5-\mathrm{kb}$ transcript, from alternative splicing of the single pre-mRNA, or, alternatively, from different genes. Based on the results obtained from DNA gel blot analysis, it seems possible that these transcripts might derive from different genes located in the cassava genome.

In response to fluctuating level of rainfall, a correlation between MeSPS and Me14-3-3 expression patterns was observed. This finding not only supports the idea that these proteins need to co-exist to form a multi-protein complex, but also suggests that the apparent levels of MeSPS and Me14-3-3 transcripts need to be tightly regulated. This type of regulation is supposedly important for both growth and survival of cassava since it allows plants to respond to the cellular energy demand that might change according to the season. In contrast to MeSPS and Me14-3-3, relatively stable levels of MeSPP transcript were observed during storage root development. However, when RT-PCR was carried out, it was observed that the levels of MeSPP transcript fluctuated slightly, particularly in storage roots of wet crop harvested at 3 months old. We therefore hypothesize that the high levels of MeSPP and MeSPS transcripts observed at this stage possibly reflects the increasing rate of sucrose (re)synthesis to supply energy derived from degradation of reserve starch.

In source organs, we observed high expression level of MeSPS in young leaves and significantly lower level in mature leaves. These results were inconsistent with Actinidia chinensis, in which no significant changes in $A c S P S$ transcript level could be observed in leaves at different developmental stages (small, expanding and senescence leaves) (Fung et al., 2003). These probably result from, for example, the diverse nature of plants, the different turnover rate of leaf sucrose and the different criteria used to identify the leaf developmental stage. Additionally, we also observed that expression of MeSPS in cassava leaves varied depending on the day/night cycle. This was particularly true for young leaves, in which a slightly higher level of MeSPS transcript was observed in leaves harvested at night. At night, leaf starch is degraded to form sucrose for storage in the vacuole and/or for transport through phloem to storage organs. During this stage, the rate of sucrose synthesis increases and this therefore explains why a slightly high level of MeSPS transcript could be observed. For MeSPP, high level of the leaf transcript was observed in young leaves, while none was detected in mature leaves. In comparison with the levels of MeSPS transcript, MeSPP was always present at a significantly lower level. Therefore, it was not surprising to detect none of the MeSPP transcript in mature cassava leaves, in which $M e S P S$ was present at relatively low level.

\section{Conclusion}

Previous studies by Pardales et al. (1996) and Sriroth et al. (2001) indicated that drought followed with onset of rain could negatively affect yield of starch in storage roots of cassava. To gain more insight into the metabolic fate of starch, the investigation of expression profiling of the three genes involved in the cassava sucrose synthesis was conducted. In conclusion, the results from this study indicated that the fluctuating levels of MeSPS, MeSPP and Me14-3-3 transcripts in sink organ, seemed to be affected to a much greater degree by different planting season than by developmental stage of the roots. Although various factors could contribute to the difference in planting season, it seems that the amount of water available to the plants has played a significant role in determining expression patterns of MeSPS, MeSPP and Me14-3-3 in storage root tissue of cassava. Given that metabolic processes are generally regulated at multiple levels, further studies need to be conducted to provide more information on MeSPS, MeSPP and Me14-3-3 isoforms, as well as their regulation at both transcriptional and post-transcriptional levels. The knowledge obtained should lead us to understand the role of these enzymes and the importance of sucrose metabolism in seasonal drought stress response in cassava. Due to the identified new role for cassava as a possible biofuel crop as well as its current usage as a food crop, further understanding on the correct time to plant and harvest cassava in order to maximize starch quantity and quality is warranted.

\section{Acknowledgements}

This research was financially supported by the National Center for Genetic Engineering and Biotechnology 
(BIOTEC) (Grant No. BT-B-09-PG-BC-4605 and BT-B-09-PG-BC-4606) and Mahidol University. KT was partially supported by the Center of Excellence for Agricultural Biotechnology, Science and Technology Postgraduate Education and Research Development Office (PERDO), Commission on Higher Education, Ministry of Education, Thailand.

\section{References}

Aravind, L., Galperin, M. Y., \& Koonin, E. V. (1998). The catalytic domain of the P-type ATPase has the haloacid dehalogenase fold. Trends in Biochemical Sciences, 23, 127-129. http://dx.doi.org/10.1016/S0968-0004(98)01189-X

Baker, S. S., Wilhelm, K. S., \& Thomashow, M. F. (1994). The 5'-region of Arabidopsis thaliana cor15a has cis-acting elements that confer cold-, drought- and ABA-regulated gene expression. Plant Molecular Biology 24, 701-713. http://dx.doi.org/10.1007/BF00029852

Bornke, F. (2005). The variable C-terminus of 14-3-3 proteins mediates isoform-specific interaction with sucrose-phosphate synthase in the yeast two-hybrid system. Journal of Plant Physiology, 162, 161-168. http://dx.doi.org/10.1016/j.jplph.2004.09.006

Castleden, C. K., Aoki, N., Gillespie, V. J., MacRae, E. A., Quick, W. P., Buchner, P., ... Lunn, J. E. (2004). Evolution and function of the sucrose-phosphate synthase gene families in wheat and other grasses. Plant Physiology 135, 1753-1764. http://dx.doi.org/10.1104/pp.104.042457

Chen, S., Hajirezaei, M., \& Bornke, F. (2005). Differential expression of sucrose-phosphate synthase isoenzymes in tobacco reflects their functional specialization during dark-governed starch mobilization in source leaves. Plant Physiology, 139, 1163-1174. http://dx.doi.org/10.1104/pp.105.069468

Church, G. M., \& Gilbert, W. (1984). Genomic sequencing. Proceeding of National Academy of Sciences USA 81, 1991-1995. http://dx.doi.org/10.1073/pnas.81.7.1991

do Nascimento, J. R. O., Cordenunsi, B. R., Lajolo, F. M., \& Alcocer, M. J. C. (1997). Banana sucrose-phosphate synthase gene expression during fruit ripening. Planta, 203, 283-288. http://dx.doi.org/10.1007/s004250050193

Echeverria, E., Salvucci, M. E., Gonzalez, P. C., Paris, G., \& Salerno, G. L. (1997). Physical and kinetic evidence for an association between sucrose-phosphate synthase and sucrose-phosphate phosphatase. Plant Physiology, 115, 223-227.

Engler-Blum, G., Meier, M., Frank, J., \& Muller, G. (1993). Reduction of background problems in non-radioactive Northern and Southern blot analyses enables higher sensitivity than ${ }^{32} \mathrm{P}$-based hybridizations. Analytical Biochemistry, 210, 235-244. http://dx.doi.org/10.1006/abio.1993.1189

Fung, R. W. M., Langenkamper, G., Gardner, R. C., \& MacRae, E. (2003). Differential expression within an SPS gene family. Plant Sciences, 164, 459-470. http://dx.doi.org/10.1016/S0168-9452(02)00430-2

Geigenberger, P., Reimholz, R., Geiger, M., Merlo, L., Canale, V., \& Stitt, M. (1997). Regulation of sucrose and starch metabolism in potato tubers in response to short-term water deficit. Planta, 201, 502-518. http://dx.doi.org/10.1007/s004250050095

Geigenberger, P., Reimholz, R., Deiting, U., Sonnewald, U., \& Stitt, M. (1999). Decreased expression of sucrose phosphate synthase strongly inhibits the water stress-induced synthesis of sucrose in growing potato tubers. Plant Journal, 19, 119-129. http://dx.doi.org/10.1046/j.1365-313X.1999.00506.x

Higo, K., Ugawa, Y., Iwamoto, M., \& Korenaga, T. (1999). Plant cis-acting regulatory DNA elements (PLACE) database. Nucleic Acids Research, 27, 297-300. http://dx.doi.org/10.1093/nar/27.1.297

Huber, J. L. A., \& Huber, S. C. (1992). Site-specific serine phosphorylation of spinach leaf sucrose-phosphate synthase. Biochemistry Journal, 283, 877-882.

Huang, J. Z., \& Huber, S. C. (2001). Phosphorylation of synthetic peptides by a CDPK and plant SNF1-related protein kinase. Influence of proline and basic amino acid residues at selected positions. Plant and Cell Physiology, 42, 1079-1087. http://dx.doi.org/10.1093/pcp/pce137

Konagaya, K., Matsushita, Y., Kasahara, M., \& Nyunoya, H. (2004). Members of 14-3-3 protein isoforms interacting with the resistance gene product $\mathrm{N}$ and the elicitor of tobacco mosaic virus. Journal of General Plant Pathology, 70, 221-231. http://dx.doi.org/10.1007/s10327-003-0113-4

Langenkamper, G., Fung, R. W. M., Newcomb, R. D., Atkinson, R. G., Gerdner, R. C., \& MacRae, E. A. (2002). 
Sucrose phosphate synthase genes in plants belong to three different families. Journal of Molecular Evolution, 54, 322-332.

Lunn, J. E., Ashton, A. R., Hatch, M. D., \& Heldt, H. W. (2000). Purification, molecular cloning, and sequence analysis of sucrose- $6^{\mathrm{F}}$-phosphate phosphohydrolase from plants. Proceeding of National Academy of Sciences USA, 97, 12914-12919. http://dx.doi.org/10.1073/pnas.230430197

Lutfiyya, L. L., Xu, N., D’Ordine, R. L., Morrell, J. A., Miller, P. W., \& Duff, S. M. G. (2007). Phylogenetic and expression analysis of sucrose phosphate synthase isozymes in plants. Journal of Plant Physiology, 167, 923-933. http://dx.doi.org/10.1016/j.jplph.2006.04.014

Mauchler-Bauer, A., Anderson, J. B., Chitsaz, F., Derbyshire, M. K., DeWeese-Scott, C., ... Bryant, S. H. (2009). CDD: specific functional annotation with the conserved domain database. Nucleic Acids Research, 37, D205-D210. http://dx.doi.org/10.1093/nar/gkn845

Moorhead, G., Douglas, P., Cotelle, V., Harthill, J., Morrice, N., Meek, S., Deiting, U., Stitt, M., Scarabel, M., Aitken, A., \& MacKintosh, C. (1999). Phosphorylation-dependent interactions between enzymes of plant $\begin{array}{llllll}\text { metabolism and } 14-3-3 & \text { proteins. Plant }\end{array}$ http://dx.doi.org/10.1046/j.1365-313X.1999.00417.x

Nguyen, T. L. T., Gheewala, S. H., \& Garivait, S. (2007). Full chain energy analysis of fuel ethanol from cassava in Thailand. Environmental Science and Technology, 41, 4135-4142. http://dx.doi.org/10.1021/es0620641

Pardales, J. R. Jr. \& Esquibel, C. B. (1996). Effect of drought during the establishment period on the root system development of cassava. Japanese Journal of Crop Sciences, 65, 93-97. http://dx.doi.org/10.1626/jcs.65.93

Quick, P., Siegl, G., Neuhaus, E., Feil, R., \& Stitt, M. (1989). Short-term water stress leads to a stimulation of sucrose synthesis by activating sucrose-phosphate synthase. Planta, 177, 535-546. http://dx.doi.org/10.1007/BF00392622

Rosenquist, M., Alsterfjord, M., Larsson, C., \& Sommarin, M. (2001). Data mining the Arabidopsis genome reveals fifteen 14-3-3 genes. Expression is demonstrated for two out of five novel genes. Plant Physiology, 127, 142-149. http://dx.doi.org/10.1104/pp.127.1.142

Santisopasri, V., Kurotjanawong, K., Chotineeranat, S., Piyachomkwan, K., Sriroth, K., \& Oates, C. G. (2001). Impact of water stress on yield and quality of cassava starch. Industrial Crops and Products, 13, 115-129. http://dx.doi.org/10.1016/S0926-6690(00)00058-3

Sehnke, P. C., Henry, R., Cline, K., \& Ferl, R. J. (2000). Interaction of a plant 14-3-3 protein with the signal peptide of a thylakoid-targeted chloroplast precursor protein and the presence of 14-3-3 isoforms in the chloroplast stroma. Plant Physiology, 122, 235-241. http://dx.doi.org/10.1104/pp.122.1.235

Sojikul, P., Kongsawadworakul, P., Viboonjun, U., Intawong, B., Narangajavana, J., \& Svasti, J. Mr. (2010). AFLP-based transcript profiling for cassava genome-wide expression analysis in the onset of storage root formation. Physiologia Plantarum, 140, 189-298. http://dx.doi.org/10.1111/j.1399-3054.2010.01389.x

Sriroth, K., Santisopasri, V., Petchalanuwat, C., Kurotjanawong, K., Piyachomkwan, K., \& Oates, C. G. (1999). Cassava starch granule structure-function properties: influence of time and conditions at harvest on four $\begin{array}{lllll}\text { cultivars of cassava starch. } & \text { Carbohydrate Polymers, } 38, & \text { 161-170. }\end{array}$ http://dx.doi.org/10.1016/S0144-8617(98)00117-9

Sriroth, K., Piyachomkwan, K., Santisopasri, V., \& Oates, C. G. (2001). Environmental conditions during root development: drought constraint on cassava starch quality. Euphytica, 120, 95-101. http://dx.doi.org/10.1023/A:1017511806128

Szopa, J., Wrobel, M., Matysiak-Kata, I., \& Swiedrych, A. (2001). The metabolic profile of the 14-3-3 repressed transgenic potato tubers. Plant Science, 161, 1075-1082. http://dx.doi.org/10.1016/S0168-9452(01)00502-7

Tonukari, N. J. (2004). Cassava and the future of starch. Electronic Journal of Biotechnology, 7, 5-8. http://dx.doi.org/10.2225/vol7-issue1-fulltext-i02

Toroser, D., Athwal, G. S., \& Huber, S. C. (1998). Site-specific regulatory interaction between spinach leaf sucrose-phosphate synthase and 14-3-3 proteins. FEBS Letters, 435, 110-114. http://dx.doi.org/10.1016/S0014-5793(98)01048-5

Winter, H., \& Huber, S. C. (2000). Regulation of sucrose metabolism in higher plants: localization and regulation of activity of key enzymes. Critical Reviews in Plant Sciences, 35, 253-289. 
Wu, K., Rooney, M. F., \& Ferl, R. J. (1997). The Arabidopsis 14-3-3 multigene family. Plant Physiology, 114, 1421-1431. http://dx.doi.org/10.1104/pp.114.4.1421

Xu, W. F., \& Shi, W. M. (2006). Expression profiling of the 14-3-3 gene family in response to salt stress and potassium and iron deficiencies in young tomato (Solanum lycopersicum) roots: analysis by real-time RT-PCR. Annals of Botany, 98, 965-974. http://dx.doi.org/10.1093/aob/mcl189

Zuk, M., Skala, J., Biernat, J., \& Szopa, J. (2003). Repression of six 14-3-3 protein isoforms resulting in the activation of nitrate and carbon fixation key enzymes from transgenic potato plants. Plant Science, 165, 731-741. http://dx.doi.org/10.1016/S0168-9452(03)00231-0 